\title{
Management of Human Resources inside Open Innovation Organizations: Some Reflections
}

\author{
Piciocchi P1 ${ }^{1}$ Clara Bassano $\mathrm{C}^{2 *}$ and Pietronudo MC $^{3}$ \\ ${ }^{1}$ Associate Professor of Management, University of Salerno, Italy \\ ${ }^{2}$ Assistant Professor of Marketing Management, Parthenope University of Naples, \\ Italy
}

${ }^{3} \mathrm{PhD}$ Student in Management, Parthenope University of Naples, Italy

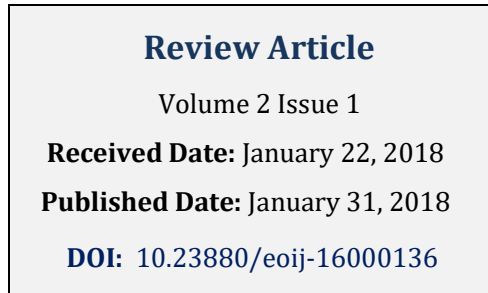

*Corresponding author: Clara Bassano, Assistant Professor of Marketing Management, Parthenope University of Naples, Italy, Email: clara.bassano@uniparthenope.it

\section{Abstract}

This paper refers to High performance HR inside organizations oriented to Open Innovation. The need for this work dates back to phenomena shifts of our more and more related market towards Open Innovation (OI). The Systems which are discussed in this study are enterprises of today which are oriented to OI, focusing on the human side of it, particularly on Human Resources Management (HRM) in order to thrive competitiveness. It deals with an overview upon the emergence of the phenomenon of Open Innovation remarking its effects on the "changing landscape of knowledge".

Nowadays enterprises find themselves inside the process of globalization which turns organizations of mere production into organizations of global production, where it is very difficult to crowd out a competition which is against local specializations and ramming of productive activities in places capable of offering specific competitive advantages. Therefore, the system of Closed Innovation is not reliable anymore: the change to Open Innovation is needed to thrive positively within the global market.

Methodological approach we used refers to Service Science: a strategic area of scientific study of Service Systems at IBM Research. Service science aims to define and improve interactions of multiple entities which work together to achieve win-win outcomes or mutual benefits. More precisely, "a service is value co-creation in the sense of a change or a set of changes which people prefer and realize as a result of their communication, planning, or other purposeful and knowledge-intensive interactions". Services are implemented by OI processes, determining change in the strategy of the company.

In this contextualized scenario, "management of Human Resources inside OI organizations" is discussed. Pointing out that employee, their knowledge, skill sets, and attitudes towards collaboration determine the effectiveness of OI strategies and practices the HR department can play a crucial role in bringing about the necessary change in attitudes. More 


\section{Ergonomics International Journal}

specifically, by developing HR practices in terms of both internal and external talent management according to the emergent T-Shaped Profile of Professionals.

Keywords: Open Innovation; Human Resources Management; T-Shaped Professionals; Service Science Management Engineering+Design

Abbreviations: HRM: Human Resources Management; OI: Open Innovation; VSA: Viable System Approach; SSMED: Service Science, Management, Engineering, and Design

\section{Introduction}

This paper refers to High performance HR inside organizations oriented to Open Innovation. The need for this work dates back to phenomena shifts of our more and more related market towards Open Innovation (OI). Like many changes, this shift reason was caused by a domino effect over the years until now. Market is dynamic and systems to survive have to find a way to adapt to it. The systems which are discussed in this study are enterprises of today which are oriented to OI, focusing on the human side of it, particularly on Human Resources Management (HRM) in order to thrive competitiveness. It deals with an overview upon the emergence of the phenomenon of Open Innovation. Henry Chesbrough has introduced this term for the first time in his book "The era of Open Innovation" (2003), remarking its effects and the "changing landscape of knowledge" [1-4].

Nowadays enterprises find themselves inside the process of globalization which turns organizations of mere production into organizations of global production, where it is very difficult to crowd out a competition which is against local specializations and ramming of productive activities in places capable of offering specific competitive advantages. Therefore, the system of Closed Innovation is not reliable anymore. In the traditional closed innovation model, the Research and Development process depends completely on internal laboratories and resources, while new ideas and technologies are investigated and presented by internal resources.

Closed Innovation relied on the creation of value based on the logics of the 'first move'. However concepts such as collaboration, networks, empathy and knowledge had not been introduced yet. Each enterprise believed that in order to develop an idea, it had to rely only on its Research and Development department or, more in general, on internal exchange of resources, stressing its boarders to be reinforced from the inside without any (or minimum) transfers with the outside. An organization was considered strong when it had full control, hierarchical development, influence strategy and valorization of its internal human capital. Hence, the change to Open Innovation was needed to thrive positively within the global market [5].

Methodological approach we used refers to Service Science a strategic area of scientific study of Service Systems at IBM Research. In a traditional point of view, services include transportation, consulting, outsourcing, retail, healthcare, entertainment, hospitality, and so on, making part for more than $80 \%$ of economic activity in the US and other industrialized countries [6]. Service science is short for Service Science, Management, Engineering, and Design, also known as SSMED [7]. It began as a 'call to action,' focusing academics, businesses, and governments on the need for research and education in areas related to service [8].

Service science aims to define and improve interactions of multiple entities which work together to achieve winwin outcomes or mutual benefits. More precisely, "a service is value co-creation in the sense of a change or a set of changes which people prefer and realize as a result of their communication, planning, or other purposeful and knowledge-intensive interactions" [9].

Services are implemented by OI processes, determining change in the strategy of the company. As Porter says in his book "Competitive Strategy", "Competitive strategy is the search for a favorable competitive position in an industry, the fundamental arena in which competition occurs $[10,11]$. Competitive strategy aims to establish a profitable and sustainable position against the forces that determine industry competition.

In this contextualized scenario, "management of Human Resources inside OI organizations" is discussed. Pointing out that employee, their knowledge, skill sets, and attitudes towards collaboration determine the effectiveness of OI strategies and practices the HR department can play a crucial role in bringing about the necessary change in attitudes. More specifically, by developing HR practices in terms of both internal and external talent management the HR department can contribute to an organizational culture where OI can thrive and all employees are rewarded for searching both 


\section{Ergonomics International Journal}

internally and beyond their departmental/organizational boundaries for new knowledge.

\section{HR Role Nowadays in the Light of Different Paradigmatic Changes}

The competitive forces that managers face today and will continue to confront in the future demand organizational excellence. The efforts to achieve such excellence through a focus on learning, quality, teamwork, and reengineering are driven by the way organizations get things done and how they treat their people. Those are fundamental HR issues. To state it plainly: achieving organizational excellence must be the work of HR.

What is necessary, hence, it is to create an entirely new role and agenda for the field that focuses it not on traditional HR activities, such as staffing and compensation, but on outcomes. HR should not be defined by what it does but by what it delivers-results that enrich the organization's value to customers, investors, and employees [12].

Regardless of their industry, size, or location, companies today face critical business challenges. Collectively, these challenges require organizations to build new capabilities. Who is currently responsible for developing those capabilities? Everyone and no one. That vacuum is HR's opportunity to play a leadership role in enabling organizations to meet the following competitive challenges:

- Globalization: With the rapid expansion of global markets, managers are struggling to balance the paradoxical demand to think globally and act locally. That imperative requires them to move people, ideas, products, and information around the world to meet local needs. They must add new and important ingredients to the mix when making strategy: volatile political situations, contentious global trade issues, fluctuating exchange rates, and unfamiliar cultures. They must be more literate in the ways of international customers, commerce, and competition than ever before. In short, globalization requires that organizations increase their ability to learn and collaborate and to manage diversity, complexity, and ambiguity.

- Profitability through Growth: During the past decade, most Western companies have been clearing debris, using downsizing, reengineering, de-layering, and consolidation to increase efficiency and cut costs. The gains of such yard work, however, have largely been realized, and executives will now have to pay attention to the other part of the profitability equation: revenue growth. The drive for revenue growth, needless to say, puts unique demands on an organization. Companies seeking to acquire new customers and develop new products must be creative and innovative, and must encourage the free flow of information and shared learning among employees. They must also become more market focused-more in touch with the fast changing and disparate needs of their customers. And companies seeking growth through mergers, acquisitions, or joint ventures require other capabilities, such as the finely honed skills needed to integrate different organizations' work processes and cultures [13].

- Technology: Technology has made our world smaller and faster. Ideas and massive amounts of information are in constant movement. The challenge for managers is to make sense and good use of what technology offers. Not all technology adds value. But technology can and will affect how and where work gets done. In the coming years, managers will need to figure out how to make technology a viable, productive part of the work setting. They will need to stay ahead of the information curve and learn to leverage information for business results. Otherwise, they risk being swallowed by a tidal wave of data-not ideas.

- Intellectual Capital: Knowledge has become a direct competitive advantage for companies selling ideas and relationships (think of professional service, software, and technology-driven companies) and an indirect competitive advantage for all companies attempting to differentiate themselves by how they serve customers. From now on, successful companies will be the ones that are the most adept at attracting, developing, and retaining individuals who can drive a global organization that is responsive to both its customers and the burgeoning opportunities of technology. Thus the challenge for organizations is making sure they have the capability to find, assimilate, develop, compensate, and retain such talented individuals.

- Change: Perhaps the greatest competitive challenge companies' face is adjusting to-indeed, embracingnonstop change. They must be able to learn rapidly and continuously, innovate ceaselessly, and take on new strategic imperatives faster and more comfortably. Constant change means organizations must create a healthy discomfort with the status quo, an ability to detect emerging trends quicker than the competition, an ability to make rapid decisions, and the ability to seek new ways of doing business. To thrive, in other words, companies will need to be in a never-ending state of transformation, perpetually creating fundamental, enduring change.

In this scenario, the role for HR as a strategic asset is to identify methods for renovating the parts of the organizational architecture that need it. In other words, HR managers should be assigned to take the lead in proposing, creating, and debating best practices in culture 


\section{Ergonomics International Journal}

change programs, for example, or in appraisal and reward systems. Similarly, if strategy implementation requires, say, a team-based organizational structure, HR would be responsible for bringing state-of-the-art approaches for creating this structure to senior management's attention.

Furthermore, HR must take stock of its own work and set clear priorities. At any given moment, the HR staff might have a dozen initiatives in its sights, such as payfor-performance, global team-work, and action-learning development experiences. But to be truly tied to business outcomes, HR needs to join forces with operating managers to systematically assess the impact and importance of each one of these initiatives.

Because becoming a strategic asset means an entirely new role for $\mathrm{HR}$, it may have to acquire new skills and capabilities. Its staff may need more education in order to perform the kind of in-depth analysis an organizational audit involves, for example. Ultimately, such new knowledge will allow HR to add value to the executive team with confidence. In time, the concept of HR as a strategic partner will make business sense.

Within the HR function are dozens of processes that can be done better, faster, and cheaper. Finding and fixing those processes is part of the work of the new HR. Some companies have already embraced these tasks, and the results are impressive. One company has created a fully automated and flexible benefits program that employees can manage without paperwork; another has used technology to screen résumés and reduce the cycle time for hiring new candidates; and a third has created an electronic bulletin board that allows employees to communicate with senior executives. In all three cases, the quality of HR work improved and costs were lowered, generally by removing steps or leveraging technology.

Work today is more demanding than ever-employees are continually being asked to do more with less. And as companies withdraw the old employment contract, which was based on security and predictable promotions, and replace it with faint promises of trust, employees respond in kind. Their relationship with the organization becomes transactional. They give their time but not much more.

\section{What are Skill Sets and why it is needed in Order to Innovate Openly?}

According to this factor, we consider the question: "What are Skill Sets and why it is needed in order to innovate openly?"

Skills Set allows an organization to know its own resources, to identify development areas, to plan training interventions and to monitor constantly degree of satisfaction and growth within the organization itself. It is very important to unify the individual perspective, in which individuals' success condition becomes central, with the strategic perspective in which the success of the enterprise is defined, so as to put in relation business, people and results. Defined and measured competences are, hence, characterized by the kind of business, by the relationship between the company and market context, by values and strategies.

Skills Set is the process through which a company relieves, manages and develops its portfolio of skills. It represents a reliable compass, based on objective evaluations of someone's skills. Skills Set is a system that ensures people-oriented management, both in terms of retribution and carrier development, by contributing to withhold the best resources as well as to allow the planning of insert actions, formation and paths for improvement. Given a skills set, it can be useful to start from the principle of Le Boterf [14] which is being competent and adequate in a certain role within an organization is knowing how to use necessary resources (i.e. skills: know, know-how, learn, act, will to act), and not only to have them.

It is possible, in fact, to have all these necessary/adequate competencies/knowledge for an excellent performance in a role, but if they cannot be activated or just partly used, it will not be possible to reach the goals set. Vice versa, a person could lack in experience and knowledge but still gain good results by activating orientation to learning skills or decisionmaking skills [15]. Skills are the core contents of the system, knowledge and technical/professional optimal/ideal capabilities a person should have and know how to use in order to assume correct behaviors that allow everyone to perform the duties and specific responsibilities requested for the position within the organization [16].

It is about a person's Adequacy to the Role and not ability in the performance, of what is necessary, what someone should know (knowledge), know-how (skills/abilities) and knowing how-to-live (behaviors/attitudes) in order to perform with personal and business success the role assigned - as known as KSA Knowledge, Skills e Abilities.

Many authors have pointed out that OI is essentially a people-driven business and that it is therefore crucial to learn how HR practices can drive Open Innovation success. It may in fact be easier to adapt organizational 


\section{Ergonomics International Journal}

processes than it is to change the attitudes of employees towards collaborating with external partners.

HR practices related to both internal and external talent management are instrumental in changing the attitudes and behaviors of employees towards Open Innovation that in turn have a significant influence on its effectiveness. Furthermore, these important HR strategies shape the organizational culture and climate that also affect the willingness of employees to engage in collaborative behavior.

It is mentioned several times in the literature that HR departments need to cultivate the right skills in employees (through selection and training) in order to stimulate their positive attitudes towards collaboration. Some of these Open-Innovation skills are related to entrepreneurship, internal and external networking, change management, learning, uncertainty tolerance, and so on. These soft skills may be more important determinants of OI success than hard skills.

Performance evaluation systems should address these soft OI skills if employees are to develop the necessary behaviors for Open Innovation. It may, for instance, not only be important to evaluate OI professionals on how effectively they can manage an OI project through the stage-gate processes but also on their abilities to successfully manage their relations with important external knowledge sources such as universities/SMEs or their willingness to learn from failed OI projects [17].

Another important HR practice related to internal talent management that may stimulate the development of OI skills in employees is referred to as "strategic talent exchange". Besides swapping employees with other companies HR departments may also encourage the exchange of personnel between internal organizational departments where it is particularly important for OI professionals to be familiarized, for example, with the R\&D department.

A final important notion related to internal talent management that can be found in the existing literature is that the dynamism inherent to OI skills should not be taken for granted. Different OI skills (present in different employees) may need to be employed in different phases of the OI process. It is thus crucial for HR departments to manage a pool of OI skills and to make these competencies accessible at the right time.

On the other hand, not much has been written on external talent management as only few organizations (most notably large consulting firms) have developed external talent management practices. These HR practices are mostly related to cultivating strong relations with external OI professionals so that they can be employed when particular OI skills or knowledge elements cannot be found internally.

The literature points out that employees, their skill sets, and attitudes towards collaboration determine the effectiveness of OI strategies and practices. Even though these soft organizational aspects are quite hard to alter, the HR department can play a crucial role in bringing about the necessary change in attitudes. More specifically, by developing HR practices in terms of both internal and external talent management the HR department can contribute to an organizational culture where OI can thrive and all employees are rewarded for searching both internally and beyond their departmental/organizational boundaries for new knowledge [17].

\section{The Character of the T-Shaped OI Professional}

An important part of the discussion revolves around the question of whether it is actually possible to identify a specific set of skills that each OI professional should have in order for them to be successful at their OI activities. In relation to this question, taking into consideration the environmental change towards Open Innovation and the character of the T-Shaped person, it is possible to discern a number of skills that are generally helpful with respect to OI there has to be a match between the person and the environment in order for the combination to generate favorable results.

So as to use these Service Science concepts in a new paradigm shift in order to innovate through knowledge creation and transfer, we can make these acknowledgments converge into the study of a new skilled profile of professionals in a new efficient way of working.

The "shape" of a professional is a term used in order to understand whether a professional is a deep specialist in one area ("I-shaped"), deep specialist in two areas ("Hshaped"), deep in just one area, but with good knowledge and communication skills across many other areas ("Tshaped"), or not deep, but with good breadth, a generalist ("Dash-shaped").

"H-shaped are also sometimes referred to as Hybrid professionals. Many applied computer scientists are $\mathrm{H}$ shaped, deep in computer science as well as some application domain such as meteorology, physics, or another area in which they build simulations or application software to perform research. If a person is deep in two 
areas, and also has good breadth for complex communication across disciplines, they are referred to as $\mathrm{Pi}$ shaped. A professional journalist is typically T-shaped, deep in communication theory as well as a versatile communicator in many topic areas. Professional science master students tend to be T-shaped with depth in one area of science, as well as with broad communications skills across many business functions" [18].

Clearly, an even more intricate shape language could be created when one factor in distinction such as rigorous theoretical knowledge ("book learning") and practical professional experience ("real-world relevance"). For our purposes, most of the points we are concerned with can be discussed in the context of the basic shapes above:

- I-Shaped people [19]: I-shaped people have a very narrow, but expert domain skills in one specific area (depth).

- H-Shaped people [18]: H-Shaped people are deep specialist in two areas (depth).

- T-Shaped people [20]: T-shaped professionals have problem-solving (depth) and complex-communication (breadth) skills.

- Pi-Shaped people [21]: Pi-Shaped (П) people have an understanding of how to use data and analytics tools (depth), but is also able to tap into a broader range of skills both in art and science, "with capabilities in both 'left brains' and 'right brain' disciplines" (breadth).

- M-Shaped people [22]: M-shaped people have multiple specialties. From the point of view of flexibility, a person with more than one specialty can be applied more flexibly than someone with single specialty. Each additional specialty shifts our mental picture from a letter to a comb.

- Dash-Shaped people [23]: A Dash-Shaped person has a wide (breadth) yet shallow base of knowledge, and no discernible specialties. This person is typically known as a generalist.

Naturally, T-shaped professional is the profile much more coherent with OI context; in fact, T-Shaped professional may be very good at collaboration, and good innovators, solving new problems in their area of depth as well.

Widely recognized SSME programs would help ensure the availability of a large population of T-shaped professionals (from many home disciplines) with the ability to collaborate to create service innovations. SSME qualifications would indicate that these graduates could communicate with scientists, engineers, managers, designers, and many others involved in service systems [24-25]. Graduates with SSME qualifications would be well prepared to 'hit the ground running', able to become immediately productive and make significant contributions when joining a service innovation project [26].

The need for T-shaped skills surfaces anywhere problem solving is required across different deep functional knowledge bases or at the juncture of such deep knowledge with an application area. People possessing these skills are able to shape their knowledge to fit the problem at hand rather than insist that their problems appear in a particular, recognizable form. Given their wide experience in applying functional knowledge, they are capable of convergent, synergistic thinking.

T-shaped skills (Figure 1, [27]) are both deep (the vertical part of the " $\mathrm{T}$ ") and broad (the horizontal part of the "T"); that is, their possessors can explore particular knowledge domains and their various applications in particular products. For example, people with T-shaped skills not only have a deep knowledge of a discipline (like ceramic materials engineering), but also know how their discipline interacts with other disciplines (such as polymer processing).

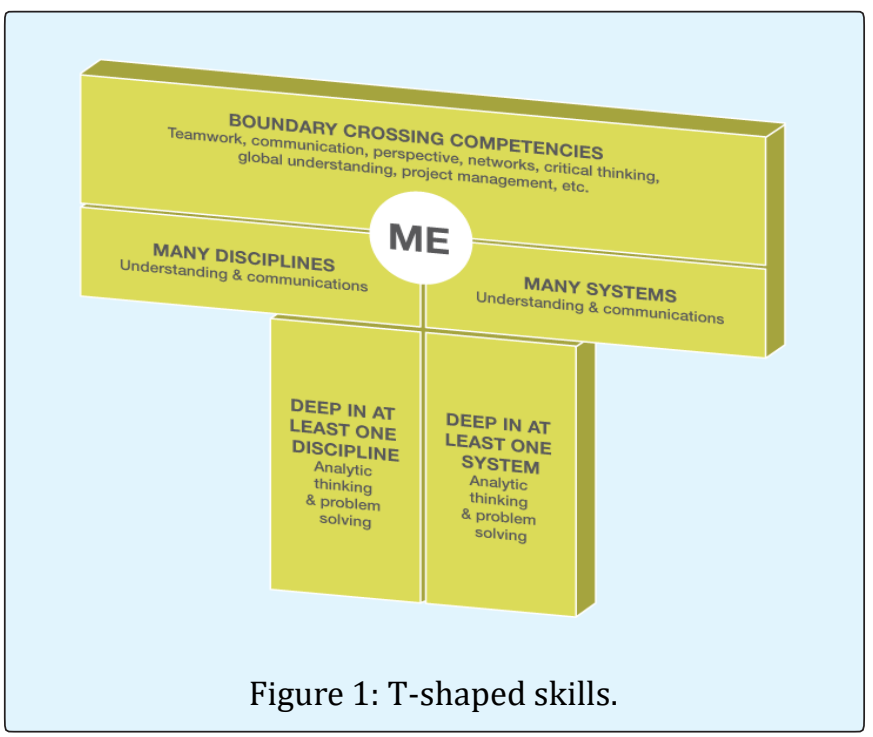

The horizontal stroke of the "T" is the disposition for collaboration across disciplines. People with T-shaped skills are extremely valuable for creating knowledge because they can integrate diverse knowledge assets. A Tshaped professional is a service scientist, with deep, expert, contributory expertise in at least one area, and broad, complex communications, and articulatory expertise across them all. They have the ability both to combine theoretical and practical knowledge and to see how their branch of knowledge interacts with other branches. Therefore, they can expand their competence across several functional branch areas, and thus create new knowledge. 


\section{Ergonomics International Journal}

T-shaped people have both depth and breadth in their skills [27]. People with T-shaped skills are extremely valuable for creating knowledge because they can integrate diverse knowledge assets. A T-shaped professional is a service scientist, with deep, expert, contributory expertise in at least one area, and broad, complex communications, and articulatory expertise across them all. They have the ability both to combine theoretical and practical knowledge and to see how their branch of knowledge interacts with other branches. Therefore, they can expand their competence across several functional branch areas, and thus create new knowledge.

One skill that is generally associated with OI success is the extent to which individuals are able to adapt to changing circumstances, i.e. their flexibility or learning potential. Research seems to suggest that flexibility tends to increase with experience: the T-Shaped Professionals indeed: "It may be that the lifelong experience of people who speak more than one language, which constantly requires shifting among tongues, explains the advantage of bilinguals in their cognitive flexibility" [28].

In general, however, it is difficult to identify the "ideal skill set" in relation to T-shaped OI professionals. It seems in fact that different skills are necessary in different phases of the Open Innovation process. Skills that are very beneficial in the early stages of innovation may be quite different from the capabilities needed in the commercialization phase. It may be unrealistic to think that all of these different skills can be found in a single individual.

Different individuals (and team compositions) may thus need to be deployed in different phases. This is one of the reasons why many large organizations have managers in charge of innovation projects who are very capable of managing start-up phases filled with ambiguity whereas managers with different skill sets take over once the project enters the commercialization stage where there is more certainty with regards to the path to follow.

Another very interesting discussion thread is related to the question of whether OI skills can be trained or only be obtained through selection. Although there seems to be general agreement that most skills can in fact be trained not all employees may be equally ready to be trained. It is therefore very important for HR departments to assess whether employees are willing to be trained before subjecting them to training sessions.

Rotating employees across organizational departments or even across firms may be more effective at cultivating
OI skills in them than traditional training programs. HR department has traditionally been a supportive/enabling function whereas for OI it is important that different organizational functions such as HR take on more proactive, strategic roles and for HR to take a lead in both internal and external talent management.

In most large organizations, the role of the HR department has already evolved in the last decade towards a more strategically oriented HR function. As is the case in most important organizational change programs the positive attitude and commitment of the top management team/HR executives heading up HR departments towards Open Innovation are crucial in bringing about the essential change in the role of the HR function in a successful manner.

This can only work in other form of organizational setting, based on decentralization, team-based organization, elimination of vertical and horizontal barriers, and development of collaborative systems, based on projects. These organizations based on projects foster the improvement of the so called co-workers' alignment, that is to say, the employees' interests and actions become aligned with organizational objectives, defined by the management, around common projects, resulting in increased productivity, satisfaction and group cohesion [29].

\section{Preliminary Conclusions}

An important part of the discussion evolves around the question of whether it is actually possible to identify a specific set of skills that each OI professional should have in order for them to be successful at their OI activities. In relation to this question, taking in consideration the environmental change towards Open Innovation and the character of the T-Shaped person, it is possible to discern a number of skills that are generally helpful with respect to OI there has to be a match between the person and the environment in order for the combination to generate favorable results.

Another very interesting discussion point is related to the question of whether OI skills can be trained or only be obtained through selection. Although there seems to be general agreement that most skills can in fact be trained not all employees may be equally ready to be trained. It is therefore very important for HR departments to assess whether employees are willing to be trained before subjecting them to training sessions.

Rotating employees across organizational departments or even across firms may be more effective at cultivating 


\section{Ergonomics International Journal}

OI skills in them than traditional training programs. In most large organizations, the role of the HR department has already evolved in the last decade towards a more strategically oriented HR function. As is the case in most important organizational change programs the positive attitude and commitment of the top management team/HR executives heading up HR departments towards Open Innovation are crucial in bringing about the essential change in the role of the HR function in a successful manner.

But, finally, our question is: How HRM should act in order to foster internal personal to being more and more relevant to the enterprise?

First, HRM should decide sets of skills a worker needs to have and define a KSA Competency Model of those skills analyzing which ones need to be implemented and how. This is so as to identify which workers are most critical to the job and work orders, because not always the best ones are the most suitable ones. Second, HRM through the Relevance Matrix [30] should discern where its workers are allocated and implement a strategy in order to foster their transversal competencies. Then, for example in this model it is thanks to the Apriori Algorithm [31], HRM should build efficient team works according to the most efficient sequence of workers, demonstrating that workers can be selected by their "frequent items".

Another implementation could be how these teams are supposed to work. As it is made for workers all these processes, this approach could be applied also to verify how team works collaborate efficiently among them in order to foster and accelerate transversal competencies.

Crossing through literature on Management of Human Resources we point out that employees, their knowledge, skill sets, and attitudes towards collaboration determine the effectiveness of OI strategies and practices. Even though these soft organizational aspects are quite hard to alter, the HR department can play a crucial role in bringing about the necessary change in attitudes. More specifically, by developing HR practices in terms of both internal and external talent management the $H R$ department can contribute to an organizational culture where OI can thrive and all employees are rewarded for searching both internally and beyond their departmental/organizational boundaries for new knowledge [32].

A new business model is needed as well, so, there are some changes about a new trend of how companies should behave within this paradigm change. We underpin an insight about the need of change of the consideration of dimensions of a company, what success, how to value create, what means to carry on a good leadership and the internal and external relationships which matter [33-36]. This new Business Model is not suitable to a hierarchical system, since OI fosters decentralization of relationships and influence inside the company whose network talents stretch from technology search to crowd facilitation and from service design to business model creation.

\section{References}

1. Chesbrough HW (2006) Open Innovation: Researching a New Paradigm. Oxford University Press.

2. Chesbrough HW (2003) Open innovation: The new imperative for creating and profiting from technology. Harvard Business Press, Boston.

3. Chesbrough HW (2004) A failing grade for the innovation academy. Financial Times.

4. Galbraith JR, Kazanjian RK (1986) Organizing to Implement Strategies of Diversity and Globalisation: The Role of Matrix Designs. Human Resource Management 25(1): 37-55.

5. Gaeta A, Gaeta M, Piciocchi P, Vollero A, Ritrovato P (2012) Exploiting Semantic Models and Techniques to Evaluate Relevance of Human Resources in Knowledge Intensive Organizations, IEE, Salerno.

6. Spohrer J, Maglio PP (2008) The emergence of service science: Toward systematic service innovations to accelerate co-creation of value. Production and Operations Management 17(3): 238-246.

7. Spohrer J, Kwan SK (2009) Service science, management, engineering, and design (SSMED): an emerging discipline-outline \& references. International Journal of Information Systems in the Service Sector 2009: 1-31.

8. Gaeta M, Perano M, Piciocchi P, Rarita L (2011) Construction of decision criteria for family-run businesses. Springer-Verlag Berlin Heidelberg.

9. Spohrer J, Maglio PP, Bailey J, Gruhl D (2007) Steps Toward a Science of Service Systems. ACMDI 40(1): 71-77.

10. Orter ME (1980) Competitive Strategy: Techniques for Analyzing Industries and Competitors. New York, Free Press. 


\section{Ergonomics International Journal}

11. Milian V (2013) Finding a Job in HR: Competency Models.

12. Procter \& Gamble (2016) Partnering for Mutual Value.

13. Powell WW (1990) Neither market nor hierarchy: network forms of organization. Research in Organizational Behaviour 12: 295-336.

14. Le Boterf G (1997) De la compétence à la navigation professionnelle, Les Éditions d'Organisation, Paris.

15. Castelli P (2012) Mappatura delle Competenze-Un Metodo e un Modello, Milano, Education.

16. McClelland DC, Spencer LM, Spencer SM (1993) Competenza nel lavoro. Modelli per una performance superiore, Milano, Franco Angeli, pp: 23-29.

17. Vanhaverbeke W, Chesbrough H, Roijakkers N (2016) Soft is Hard to Change: The Challenge of aligning HR with Open Innovation. Innovation Management, Hasselt University, Hasselt, Belgium.

18. Donofrio N, Sanchez C, Spohrer J (2008) Collaborative Innovation and Service Systems: Implications for Institutions and Disciplines. Holistic Engineering, IBM.

19. Meier JD (2013) E-Shaped People, Not T-Shaped, Agile.

20. Donofrio N, Spohrer J, Zadeh HS (2010) ResearchDriven Medical Education and Practice: A Case for TShaped Professionals. IBM.

21. Ward C (2014) More 'pi-shaped people' needed for the retail sector. MyCustomer.

22. AgileTM (2016) Productive Agile Teams: I, T, E and M Shaped People. Software process and Management.

23. Lee M (2011) T-Shaped Skills, I-Shaped Skills and Dash-Shaped Skills. BizThoughts: Thoughts on business, startups, and technology.

24. Maglio PP, Vargo SL, Caswell N, Spohrer J (2009) The Service System is the Basic Abstraction of Service Science. Information Systems and e-business Management.
25. Maglio PP (2014) Smart Service Systems. Service Science. Informs PubsOnline.

26. IfM and IBM (2008) Succeeding through service innovation: A service perspective for education, research, business and government, University of Cambridge Institute for Manufacturing.

27. Brown T (2010) T-Shaped Stars: The Backbone of IDEO's Collaborative Culture. IDEO.

28. Siegel-itzkovich J (2003) U of Haifa study: Bilingual people exhibit greater cognitive flexibility. Tel Aviv, Israel.

29. Cardoso J, de Sousa F, Pellissier R, Monteiro I (2012) Creativity, Innovation and collaborative organizations. The International Journal of Organizational Innovation 5(1): 26-24.

30. Golinelli G (2010) Viable System Approach (VSA), Governing Business Dynamics. International Journal of Systems and Society 2(2): 88-95.

31. Agrawal R, Srikant R (1994) Fast Algorithms for Mining Association Rules, IBM Almaden Research Center. 650 Harry Road, San Jose, CA 95120, pp: 1-13.

32. Leonard-Barton D (1995) Wellsprings of Knowledge: Building and Sustaining the Sources of Innovation, Harvard Business School Press.

33. Vargo SL, Maglio PP, Akaka MA (2008) On value and value co-creation: a service systems and service logic perspective. European Management Journal 26(3): 145-152.

34. Vargo S, Lusch RF (2008) Service-Dominant Logic: Continuing the Evolution. Journal of the Academy of Marketing Science 36(1): 1-10.

35. Spohrer J, Maglio PP (2009) Service Science: Toward a Smarter Planet. In Service Engineering, In: Karwowski, Salvendy (Eds.), Wiley, New York.

36. Spohrer J, Golinelli GM, Piciocchi P, Bassano C (2009) An Integrated SS-VSA Analysis of Changing Job Roles. Service Science 12(1-2): 1-20. 